\title{
Spondyloepiphyseal dysplasia, MacDermot type
}

INSERM

\section{Source}

INSERM. (1999). Orphanet: an online rare disease and orphan drug data base.

Spondyloepiphyseal dysplasia, MacDermot type. ORPHA:163668

Spondyloepiphyseal dysplasia (SED), MacDermot type is characterized by short stature, femoral epiphyseal dysplasia, mild vertebral changes and sensorineural deafness. 